\title{
Erdős-Ko-Rado theorems for uniform set-partition systems
}

\author{
Karen Meagher \\ Department of Mathematics and Statistics \\ University of Ottawa, Ottawa, Ontario, Canada \\ kmeagher@site.uottawa.ca \\ Lucia Moura \\ School of Information Technology and Engineering \\ University of Ottawa, Ottawa, Ontario, Canada \\ lucia@site.uottawa.ca
}

Submitted: Apr 29, 2005; Accepted: Jun 21, 2005; Published: Aug 25, 2005

Mathematics Subject Classifications: 05D05

\begin{abstract}
Two set partitions of an $n$-set are said to $t$-intersect if they have $t$ classes in common. A $k$-partition is a set partition with $k$ classes and a $k$-partition is said to be uniform if every class has the same cardinality $c=n / k$. In this paper, we prove a higher order generalization of the Erdös-Ko-Rado theorem for systems of pairwise $t$-intersecting uniform $k$-partitions of an $n$-set. We prove that for $n$ large enough, any such system contains at most $\frac{1}{(k-t) !}\left(\begin{array}{c}n-t c \\ c\end{array}\right)\left(\begin{array}{c}n-(t+1) c \\ c\end{array}\right) \cdots\left(\begin{array}{c}n-(k-1) c \\ c\end{array}\right)$ partitions and this bound is only attained by a trivially $t$-intersecting system. We also prove that for $t=1$, the result is valid for all $n$. We conclude with some conjectures on this and other types of intersecting partition systems.
\end{abstract}

Keywords: Erdős-Ko-Rado theorems of higher order, intersecting set partitions.

\section{Introduction}

In this paper, we prove two Erdős-Ko-Rado type theorems for systems of uniform set partitions. They are stated after some notation and background results are introduced.

For $i, j \in N, i \leq j$, let $[i, j]$ denote the set $\{i, i+1, \ldots, j\}$. For $k, n \in N$, set $\left(\begin{array}{c}{[n]} \\ k\end{array}\right)=\{A \subseteq[1, n]:|A|=k\}$. A system $\mathcal{A}$ of subsets of $[1, n]$ is said to be $k$-uniform if $\mathcal{A} \subseteq\left(\begin{array}{c}{[n]} \\ k\end{array}\right)$. A set system $\mathcal{A} \subseteq\left(\begin{array}{c}{[n]} \\ k\end{array}\right)$ is said to be $t$-intersecting if $\left|A_{1} \cap A_{2}\right| \geq t$, for all $A_{1}, A_{2} \in \mathcal{A}$. We say that $\mathcal{A} \subseteq\left(\begin{array}{c}{[n]} \\ k\end{array}\right)$ is a trivially t-intersecting set system if $\mathcal{A}$ is equal up 
to permutations on $[1, n]$ to

$$
\mathcal{A}(n, k, t)=\left\{A \in\left(\begin{array}{c}
{[n]} \\
k
\end{array}\right):[1, t] \subseteq A\right\} .
$$

The Erdős-Ko-Rado theorem [5] is concerned with the maximal cardinality of $k$ uniform $t$-intersecting set systems as well as with the structure of such maximal systems.

Theorem EKR [5] Let $n \geq k \geq t \geq 1$, and let $\mathcal{A} \subseteq\left(\begin{array}{c}{[n]} \\ k\end{array}\right)$ be a t-intersecting set system. If $n \geq(k-t+1)(t+1)$, then $|\mathcal{A}| \leq\left(\begin{array}{l}n-t \\ k-t\end{array}\right)$. Moreover, if $n>(k-t+1)(t+1)$, then this bound is tight if and only if $\mathcal{A}$ is a trivially t-intersecting set system.

The exact bound for $n$, given in the above theorem, was proven by Frankl [7] for $t \geq 15$ and by Wilson [12] for general $t$. For more information on the Erdős-Ko-Rado theorem, see [4].

Higher order extremal problems are extremal problems in which the elements in the system are set systems (called clouds) rather than sets. Ahlswede, Cai and Zhang [2] give a good overview of such problems. Most problems considered in [2] require that the clouds be pairwise disjoint. The direct generalization of the Erdös-Ko-Rado theorem for disjoint clouds proved to be false [1]. P. L. Erdős and Székely [6] survey higher order Erdős-KoRado theorems where clouds are substituted by set systems with additional structure and the disjointness requirement for pairs of set systems is dropped. They consider, among other cases, the particular case in which each structure is a set partition.

A set partition of $[1, n]$ is a set of disjoint non-empty subsets (called classes) of $[1, n]$ whose union is $[1, n]$. Throughout this paper, we refer to set partitions as simply partitions. A partition $P$ is called a $k$-partition if it contains $k$ classes, that is, $|P|=k$. Denote by $P_{k}^{n}$ the set of all $k$-partitions of $[1, n]$. A partition $P \in P_{k}^{n}$ is said to be uniform if every class of $P$ has the same cardinality, that is, $|A|=n / k$, for all $A \in P$. Denote by $U_{k}^{n}$ the set of all uniform partitions in $P_{k}^{n}$. Let $S(n, k)$ denote the Stirling number of the second type, that is $S(n, k)=\left|P_{k}^{n}\right|$. Analogously, denote $U(n, k)=\left|U_{k}^{n}\right|$. It is easy to see that for $n=c k$, we get

$$
U(n, k)=\frac{1}{k !}\left(\begin{array}{l}
n \\
c
\end{array}\right)\left(\begin{array}{c}
n-c \\
c
\end{array}\right) \cdots\left(\begin{array}{c}
n-(k-1) c \\
c
\end{array}\right),
$$

and for the trivial cases, $U(0,0)=1$ and $U(n, 0)=0$ for $n>0$.

A partition system $\mathcal{P} \subseteq P_{k}^{n}$ is said to be $t$-intersecting if $\left|P_{1} \cap P_{2}\right| \geq t$, for all $P_{1}, P_{2} \in \mathcal{P}$. So, two partitions are $t$-intersecting if they have at least $t$ classes in common. We say that $\mathcal{P} \subseteq P_{k}^{n}$ is a trivially $t$-intersecting partition system if $\mathcal{P}$ is equal up to permutations on $[1, n]$ to

$$
\mathcal{Q}(n, k, t)=\left\{P \in P_{k}^{n}:\{\{1\},\{2\}, \ldots,\{t\}\} \subseteq P\right\} .
$$

We say that $\mathcal{P} \subseteq U_{k}^{n}$ is a trivially t-intersecting uniform partition system if $\mathcal{P}$ is equal up to permutations on $[1, n]$ to

$$
\begin{aligned}
\mathcal{P}(n, k, t) \quad & \left\{P \in U_{k}^{n}:\right. \\
& \{[1, c],[c+1,2 c], \ldots,[(t-1) c+1, t c]\} \subseteq P\} .
\end{aligned}
$$


P. L. Erdős and Székely observe that the following Erdős-Ko-Rado type result for $t$-intersecting partition systems holds.

Theorem ES [6] Let $n \geq k \geq t \geq 1$, and let $\mathcal{P} \subseteq P_{k}^{n}$ be a t-intersecting partition system. If $n \geq n_{0}(k, t)$, then $|\mathcal{P}| \leq S(n-t, k-t)$. This bound is attained by a trivially t-intersecting partition system.

We prove analogous theorems for uniform partition systems that guarantee the uniqueness of the maximal system. Our first theorem completely settles the case $t=1$.

Theorem 1. Let $n \geq k \geq 1$, and let $\mathcal{P} \subseteq U_{k}^{n}$ be a 1-intersecting uniform partition system. Let $c=n / k$ be the size of a class in each partition. Then, $|\mathcal{P}| \leq U(n-c, k-1)$. Moreover, this bound is tight if and only if $\mathcal{P}$ is a trivially 1-intersecting uniform partition system.

Our second theorem deals with general $t$ and determines the cardinality and structure of maximal $t$-intersecting uniform partition systems when $n$ is sufficiently large. In this theorem, $n$ can be sufficiently large with respect to $k$ and $t$ or if $c \geq t+2$ with respect to $c$ and $t$.

Theorem 2. Let $n \geq k \geq t \geq 1$, and let $\mathcal{P} \subseteq U_{k}^{n}$ be a t-intersecting uniform partition system. Let $c=n / k$ be the size of a class in each partition. If $\left(n \geq n_{0}(k, t)\right)$ or $(c \geq t+2$ and $\left.n \geq n_{1}(c, t)\right)$ then,

1. $|\mathcal{P}| \leq U(n-t c, k-t)$;

2. moreover, this bound is tight if and only if $\mathcal{P}$ is a trivially t-intersecting uniform partition system.

When we set $c=2$, our theorems determine a maximal family of 1-regular graphs that pairwise intersect on at least $t$ edges. This is related to graph problems studied by Simonovits and Sós $[9,10,11]$ where maximal families of general graphs that intersect on a specified type of subgraph are considered. For $c>2$, our theorems determine the maximal family of 1-regular $c$-uniform hypergraphs that intersect in at least $t$ edges.

In Section 2, we give a straightforward lemma from which we can easily prove Theorem 1 for all cases except $c=2$ and Theorem 2. Indeed, the proof of Theorem 1 for $c=2$ is the only more involved case. Since this proof applies to all $c$, it is presented in this generality in Section 3. In Section 4, we mention a generalization of the concepts and results for when $c$ does not divide $n$, which naturally arises from looking at each partition as a maximal matching on a complete uniform hypergraph. In Section 5, we discuss conjectures which include other types of intersecting partition systems.

In the proofs of Lemmas 3-6 in the following sections, we apply a version of the kernel method introduced by Hajnal and Rothschild [8].

\section{Proof of Theorem 1 for $c \neq 2$ and Theorem 2}

A blocking set $\mathcal{B} \subset\left(\begin{array}{c}{[n]} \\ c\end{array}\right)$ for a uniform partition system $\mathcal{P} \subseteq U_{k}^{n}$ is a set of $c$-sets, with $c=n / k$, such that $|\mathcal{B} \cap P| \geq 1$, for all $P \in \mathcal{P}$.

Let $\mathcal{P} \subseteq U_{k}^{n}, c=n / k$ and let $A$ be a $c$-set of $[1, n]$. We denote $\mathcal{P}_{A}=\{P \in \mathcal{P}: A \in P\}$. 
Lemma 3. Let $n \geq k$ and $k-2 \geq t \geq 1$, and let $\mathcal{P} \subseteq U_{k}^{n}$ be a $t$-intersecting partition system. Let $c=n / k$ be the size of a class in each partition. Assume that there does not exist a c-set that occurs as a class in every partition in $\mathcal{P}$. Then,

$$
|\mathcal{P}| \leq k\left(\begin{array}{c}
k-2 \\
t
\end{array}\right) U(n-(t+1) c, k-(t+1)) .
$$

Proof. Let $A$ be a class from a partition in $\mathcal{P}$. Since no single class occurs in every partition in $\mathcal{P}$, there is a partition $Q \in \mathcal{P}$ that does not contain $A$. Every partition in $\mathcal{P}_{A}$ must $t$-intersect $Q$. There are at most $k-2$ classes in $Q$ that do not contain an element in $A$. Each partition in $\mathcal{P}_{A}$ must contain at least $t$ of these $k-2$ classes. Thus, for any class $A$, $\left|\mathcal{P}_{A}\right| \leq\left(\begin{array}{c}k-2 \\ t\end{array}\right) U(n-(t+1) c, k-(t+1))$.

Let $R \in \mathcal{P}$. Then, $R$ is a blocking set of $\mathcal{P}$, and $\mathcal{P}=\cup_{A \in R} \mathcal{P}_{A}$. Thus, since $|R|=k$, we get

$$
|\mathcal{P}| \leq k\left(\begin{array}{c}
k-2 \\
t
\end{array}\right) U(n-(t+1) c, k-(t+1)) .
$$

Proof of Theorem 1 for $c \neq 2$. The theorem is clearly true when $c=1$ and when $k=1$ or $k=2$. Let $n \geq k \geq 3$ and $c=n / k \geq 3$. Let $\mathcal{P} \subseteq U_{k}^{n}$ be a maximal 1-intersecting uniform partition system that is not trivially 1-intersecting. By Lemma 3

$$
|\mathcal{P}| \leq k(k-2) U(n-2 c, k-2) .
$$

For $k \geq 3$ and $c \geq 3$,

$$
\left(\begin{array}{c}
k c-c \\
c
\end{array}\right) \geq\left(\begin{array}{c}
3 k-3 \\
3
\end{array}\right) \geq k(k-1)(k-2) .
$$

Thus, we have $k(k-2)<\frac{1}{k-1}\left(\begin{array}{c}n-c \\ c\end{array}\right)$ and the theorem holds for $c \neq 2$.

Proof of Theorem 2. If $t=k$ or $t=k-1$ then two $k$-partitions are $t$-intersecting if and only if they are identical. Thus the theorem holds for $t=k$ or $t=k-1$.

Let $n \geq k$ and $k-2 \geq t \geq 1$. Let $\mathcal{P} \subseteq U_{k}^{n}$ be a maximal $t$-intersecting uniform partition system that is not trivially $t$-intersecting. Let $c=n / k$ be the size of a class in each partition. It is enough to show that for $n$ large enough $|\mathcal{P}|<U(n-t c, k-t)$.

For $c=1$, there is only one partition and $|\mathcal{P}|=1$, so we assume $c \geq 2$. Let $\mathcal{A}$ be the set of all $c$-sets that occur in every partition in $\mathcal{P}$. Let $s=|\mathcal{A}|$ and since $\mathcal{P}$ is not trivially $t$-intersecting, we have $0 \leq s<t$. Consider the system $\mathcal{P}^{\prime}=\{P \backslash \mathcal{A}: P \in \mathcal{P}\}$. The system $\mathcal{P}^{\prime}$ is a $t^{\prime}$-intersecting partition system contained in $U_{k^{\prime}}^{n^{\prime}}$, with $k^{\prime}=k-s$, 
$t^{\prime}=t-s$ and $n^{\prime}=n-s c=c(k-s)$, and $|\mathcal{P}|=\left|\mathcal{P}^{\prime}\right|$. Furthermore, there exists no $c$-set in every partition in $\mathcal{P}^{\prime}$, so by Lemma 3 ,

$$
\begin{aligned}
\left|\mathcal{P}^{\prime}\right| & \leq k^{\prime}\left(\begin{array}{c}
k^{\prime}-2 \\
t^{\prime}
\end{array}\right) U\left(n^{\prime}-\left(t^{\prime}+1\right) c, k^{\prime}-\left(t^{\prime}+1\right)\right) \\
& =(k-s)\left(\begin{array}{c}
k-s-2 \\
t-s
\end{array}\right) U(n-(t+1) c, k-(t+1)) \\
& \leq k\left(\begin{array}{c}
k-2 \\
t
\end{array}\right) U(n-(t+1) c, k-(t+1)) .
\end{aligned}
$$

For $\left(c \geq t+2\right.$ and $\left.n \geq n_{1}(c, t)\right)$ or $\left(n \geq n_{0}(k, t)\right)$, we have

$$
k\left(\begin{array}{c}
k-2 \\
t
\end{array}\right)<\frac{1}{k-t}\left(\begin{array}{c}
n-t c \\
c
\end{array}\right) .
$$

Therefore,

$$
\begin{aligned}
\left|\mathcal{P}^{\prime}\right| & <\frac{1}{k-t}\left(\begin{array}{c}
n-t c \\
c
\end{array}\right) U(n-(t+1) c, k-(t+1)) \\
& =U(n-t c, k-t) .
\end{aligned}
$$

\section{Proof of Theorem 1 for general $c$}

It only remains to prove the case $c=2$ of Theorem 1 , but we give the proof for general $c$, as it follows similarly.

Let $\mathcal{P} \subseteq U_{k}^{n}, c=n / k$ and let $\mathcal{A}$ be a set of $c$-subsets of $[1, n]$. We denote $\mathcal{P}_{\mathcal{A}}=\{P \in$ $\mathcal{P}: \mathcal{A} \subseteq P\}$.

Lemma 4. Let $n \geq k \geq 1$, and let $\mathcal{P} \subseteq U_{k}^{n}$ be a 1-intersecting partition system that is not trivially 1-intersecting. Let $c=n / k$ be the size of a class in each partition. Let $l$ be the size of the smallest blocking set for $\mathcal{P}$. Then, for any $1 \leq i<l$, any given set of $i$ classes of a partition can occur together in at most

$$
(k-i)(k-(i+1)) \cdots(k-(l-1)) U(c(k-l), k-l)
$$

partitions in $\mathcal{P}$.

Proof. Use induction on $l-i$. If $i=l-1$, consider a set of $(l-1)$ disjoint $c$-sets $\mathcal{A}=\left\{A_{1}, A_{2}, \ldots, A_{l-1}\right\}$. Since $|\mathcal{A}|<l$, the set $\mathcal{A}$ is not a blocking set for $\mathcal{P}$. So, there exists a partition $Q$ in $\mathcal{P}$ that does not contain any of the $A_{j} \in \mathcal{A}$. There are at least $l-1$ classes in $Q$ that contain some element of $A_{1} \cup A_{2} \cup \cdots \cup A_{l-1}$. So, there are at most 
$k-(l-1)$ classes in $Q$ that could appear in a partition in $\mathcal{P}_{\mathcal{A}}$. Each partition in $\mathcal{P}_{\mathcal{A}}$ must contain at least one of these $k-(l-1)$ classes. Thus,

$$
\begin{aligned}
\left|\mathcal{P}_{\mathcal{A}}\right| & \leq(k-(l-1)) U(n-(l-1) c-c, k-(l-1)-1) \\
& =(k-l+1) U(n-l c, k-l) .
\end{aligned}
$$

This completes the case $l=i+1$.

Now, for $l \geq i+1$, we assume that any set of $i$ disjoint $c$-sets can occur together in at most

$$
(k-i)(k-(i+1)) \cdots(k-l+1) U(n-l c, k-l)
$$

partitions in $\mathcal{P}$. Consider any set of $(i-1)$ disjoint $c$-sets $\mathcal{A}=\left\{A_{1}, A_{2}, \ldots, A_{i-1}\right\}$. Since $i-1<l$, there exists a partition $Q \in \mathcal{P}$, which does not contain any of the $A_{j} \in \mathcal{A}$. There are at most $k-(i-1)$ classes in $Q$ that could appear in a partition in $\mathcal{P}_{\mathcal{A}}$. By the induction hypothesis, each of these $k-(i-1)$ classes can occur together with all $A_{j} \in \mathcal{A}$ in at most $(k-i)(k-i+1) \cdots(k-l+1) U(n-l c, k-l)$ partitions. Thus,

$$
\left|\mathcal{P}_{\mathcal{A}}\right| \leq(k-(i-1))(k-i) \cdots(k-l+1) U(n-l c, k-l) .
$$

We need a slightly stronger version of the previous lemma for $i=1$.

Lemma 5. Let $n \geq k \geq 1$, and let $\mathcal{P} \subseteq U_{k}^{n}$ be a 1-intersecting system that is not trivially 1 -intersecting. Let $c=n / k$ be the size of a class in each partition. Let $l<k$ be the size of the smallest blocking set for $\mathcal{P}$. Any class can occur in at most

$$
(k-2)\left(\prod_{i=2}^{l-1}(k-i)\right) U(n-l c, k-l)
$$

partitions in $\mathcal{P}$.

Proof. From Lemma 4 with $i=2$, any pair of classes can occur in at most $(k-2) \cdots(k-$ $(l-1)) U(n-l c, k-l)$ partitions.

Let $A$ be a class in a partition in $\mathcal{P}$. Since the system is not trivially 1-intersecting, there exists a partition $Q \in \mathcal{P}$ which does not contain $A$. Any partition in $\mathcal{P}_{A}$ must intersect $Q$. The elements from $A$ must be in at least two separate classes in $Q$, thus there are at most $k-2$ classes in $Q$ which could be in this intersection. Each of these $k-2$ classes can occur in at most $(k-2) \cdots(k-(l-1)) U(n-l c, k-l)$ partitions in $\mathcal{P}_{A}$. Thus,

$$
\left|\mathcal{P}_{A}\right| \leq(k-2)\left(\prod_{i=2}^{l-1}(k-i)\right) U(n-l c, k-l)
$$


Lemma 6. Let $n \geq k \geq 4$, and let $\mathcal{P} \subseteq U_{k}^{n}$ be a 1-intersecting partition system that is not trivially 1-intersecting. Let $l$ be the size of the smallest blocking set for $\mathcal{P}$. If $l=k-1$ or $k$, then any set of $i<k-2$ classes of a partition can occur in at most $(k-i)(k-i-1)(k-i-2) \cdots 3$ partitions in $\mathcal{P}$.

Proof. Since $l \geq k-1$, for any set $\mathcal{A}$ of $(k-2)$ classes, there exists a partition $Q \in \mathcal{P}$ that does not contain any of the classes in $\mathcal{A}$. Any partition in $\mathcal{P}_{\mathcal{A}}$ must intersect $Q$ and there are at most 2 classes in $Q$ which could be in this intersection. Once a class from $Q$ is chosen, the last class of the partition is determined. Thus, any set of $k-2$ classes can occur in at most one partition in $\mathcal{P}$.

We will use induction on $k-i$. If $i=k-3$ consider a set $\mathcal{A}$ of $k-3$ classes. Since $|\mathcal{A}|<l-1$, there is a partition $Q \in \mathcal{P}$ that does not contain any of the classes in $\mathcal{A}$. There are at most $k-(k-3)=3$ classes in $Q$ that could appear in a partition in $\mathcal{P}_{\mathcal{A}}$. Since no set of $(k-2) c$-sets can occur in more than one partition, $\left|\mathcal{P}_{\mathcal{A}}\right| \leq 3$.

Now, if $i \leq k-3$ we assume that any set of $i$ classes of a partition can occur together in at most $(k-i)(k-i-1)(k-i-2) \cdots 3$ partitions in $\mathcal{P}$. Consider any set $\mathcal{A}$ of $(i-1)$ classes. There exists a partition $Q \in \mathcal{P}$ which does not contain any of the classes in $\mathcal{A}$. There are at most $k-(i-1)$ classes in $Q$ which could occur in a partition in $\mathcal{P}_{\mathcal{A}}$. Thus, $\left|\mathcal{P}_{\mathcal{A}}\right| \leq(k-(i-1))(k-i)(k-i-1)(k-i-2) \cdots 3$.

Before giving the proof of the Theorem 1, we state two lemmas, which can be easily proved by induction on $l$ and $j$, respectively.

Lemma 7. For $k-2 \geq l \geq 2$,

$$
l(k-2) \prod_{i=2}^{l-1}(k-i)<\prod_{i=1}^{l-1}(2(k-i)-1) .
$$

Lemma 8. Let $k>j, c \geq 1$ and $n=c k$. Then,

$$
U(c(k-1), k-1)=\left(\prod_{i=1}^{j-1}\left(\begin{array}{c}
c k-i c-1 \\
c-1
\end{array}\right)\right) U(c(k-j), k-j) .
$$

Proof of Theorem 1. Let $\mathcal{P} \subseteq U_{k}^{n}$ be a maximal 1-intersecting partition system that is not trivially 1-intersecting. It is enough to show that $|\mathcal{P}|<U(n-c, k-1)$. If $k=2$ or 3 then every maximal 1-intersecting partition system is trivially 1-intersecting, so we know that $k \geq 4$. For the same reason, we know $c \geq 2$. Let $l$ be the size of the smallest blocking set for $\mathcal{P}$. Since $\mathcal{P}$ is not trivially 1 -intersecting, we know that $l>1$.

Case 1. $l \leq k-2$.

There exists a blocking set $\mathcal{B}$ for $\mathcal{P}$ with $|\mathcal{B}|=l$, and from Lemma 5 each class in $\mathcal{B}$ can be in at most $(k-2)\left(\prod_{i=2}^{l-1}(k-i)\right) U(c(k-l), k-l)$ partitions in $\mathcal{P}$. Thus,

$$
|\mathcal{P}| \leq l(k-2)\left(\prod_{i=2}^{l-1}(k-i)\right) U(c(k-l), k-l) .
$$


From Lemma 7 , and the fact that $2(k-i)-2 \leq\left(\begin{array}{c}c(k-i)-1 \\ c-1\end{array}\right)$ for all $c \geq 2$ we get,

$$
l(k-2) \prod_{i=2}^{l-1}(k-i)<\prod_{i=1}^{l-1}(2(k-i)-1) \leq \prod_{i=1}^{l-1}\left(\begin{array}{c}
c(k-i)-1 \\
c-1
\end{array}\right) .
$$

Therefore,

$$
\begin{aligned}
|\mathcal{P}| & \leq l(k-2)\left(\prod_{i=2}^{l-1}(k-i)\right) U(c(k-l), k-l) & & \text { (by equation 2) } \\
& <\left(\prod_{i=1}^{l-1}\left(\begin{array}{c}
c(k-i)-1 \\
c-1
\end{array}\right)\right) U(c(k-l), k-l) & & \text { (by equation 3) } \\
& =U(c(k-1), k-1) & & \text { (by Lemma } 8 \text { with } j=l) \\
& =U(n-c, k-1) . & &
\end{aligned}
$$

Case 2. $k \geq l \geq k-1$.

By Lemma 6 , any single class can occur in at most $(k-1)(k-2)(k-3) \cdots 3$ partitions in $\mathcal{P}$. Since there exists a blocking set of cardinality $k$, then

$$
|\mathcal{P}| \leq k(k-1)(k-2)(k-3) \cdots 3=\prod_{i=1}^{k-2}(k-i+1) .
$$

We have

$$
k-i+1<2 k-2 i-1, \quad \text { for all } i \leq k-3,
$$

and

$$
2(k-i)-1 \leq\left(\begin{array}{c}
c(k-i)-1 \\
c-1
\end{array}\right), \quad \text { for all } c \geq 2 .
$$

Therefore,

$$
\begin{aligned}
& \left.|\mathcal{P}| \leq \prod_{i=1}^{k-2}(k-i+1) \quad \text { (by equation } 4\right) \\
& =3 \prod_{i=1}^{k-3}(k-i+1) \\
& <3 \prod_{i=1}^{k-3}(2 k-2 i-1) \quad(\text { by equation } 5 \text { and } k \geq 4) \\
& =\prod_{i=1}^{k-2}(2 k-2 i-1) \\
& \leq \prod_{i=1}^{k-2}\left(\begin{array}{c}
c(k-i)-1 \\
c-1
\end{array}\right) \quad \text { (by equation } 6 \text { and } c \geq 2 \text { ) } \\
& =U(c(k-1), k-1) \quad(\text { by Lemma } 8 \text { with } j=k-1 \text { and } U(c, 1)=1) \\
& =U(n-c, k-1) \text {. }
\end{aligned}
$$




\section{Intersecting Maximal Matchings}

As mentioned in the introduction, our theorems can be seen as results on maximal families of 1-regular $c$-uniform hypergraphs on $n$ vertices that intersect in at least $t$ edges. Alternatively, these hypergraphs can be thought of as perfect matchings on $K_{n}^{c}$, the complete $c$-uniform hypergraph on $n$ vertices. Thus, we can generalize our results for the case when $c$ does not divide $n$, by considering maximal matchings in place of perfect ones.

Define a $(n, c)$-packing to be a set of disjoint $c$-sets of an $n$-set. Let $P_{n, c}$ denote the set of all maximal $(n, c)$-packings, that is, all $(n, c)$-packings with $\left\lfloor\frac{n}{c}\right\rfloor c$-sets. Set $P(n, c)=\left|P_{n, c}\right|$, then for $k=\left\lfloor\frac{n}{c}\right\rfloor$,

$$
P(n, c)=\frac{1}{k !}\left(\begin{array}{l}
n \\
c
\end{array}\right)\left(\begin{array}{c}
n-c \\
c
\end{array}\right) \cdots\left(\begin{array}{c}
n-(k-1) c \\
c
\end{array}\right) .
$$

An $(n, c)$-packing system $\mathcal{P} \subseteq \mathcal{P}_{n, c}$ is $t$-intersecting if $|P \cap Q| \geq t$, for all $P, Q \in \mathcal{P}$. It is straightforward to define a trivially intersecting $t$-intersecting $(n, c)$-packing system.

Generalizations of Theorems 1 and 2 are stated next without proof. The proofs for these are very similar to the ones used for the original theorems. Indeed, the only change to the original proofs is that Lemma 3 needs to have $k-1$ in place of $k-2$ in the upper bound on $|\mathcal{P}|$.

Lemma 9. Let $n, c, t$ and $k$ be positive integers with $n \geq c$ and $t<k=\lfloor n / c\rfloor$. Let $\mathcal{P} \subseteq P_{n, c}$ be a t-intersecting $(n, c)$-packing system. Assume that there does not exist a $c$-set that occurs in every $(n, c)$-packing in $\mathcal{P}$. Then,

$$
|\mathcal{P}| \leq k\left(\begin{array}{c}
k-1 \\
t
\end{array}\right) P(n-(t+1) c, c) .
$$

Theorem 10. Let $n \geq c$ and $k=\left\lfloor\frac{n}{c}\right\rfloor$. Let $\mathcal{P} \subseteq P_{n, c}$ be a 1-intersecting $(n, c)$-packing system. Then $|\mathcal{P}| \leq P(n-c, c)$. Moreover, this bound is tight if and only if $\mathcal{P}$ is a trivially 1-intersecting $(n, c)$-packing system.

Theorem 11. Let $n \geq c$ and $t \leq k=\left\lfloor\frac{n}{c}\right\rfloor$. Let $\mathcal{P} \subseteq P_{n, c}$ be a t-intersecting $(n, c)$-packing system. If $\left(n \geq n_{0}(k, t)\right)$ or $\left(c \geq t+2\right.$ and $\left.n \geq n_{1}(c, t)\right)$ then,

1. $|\mathcal{P}| \leq P(n-t c, c)$;

2. moreover, this bound is tight if and only if $\mathcal{P}$ is a trivially t-intersecting $(n, c)$-packing system.

\section{Open problems and conjectures}

We conclude with some open problems and conjectures. The first subsection involves extensions of Theorem 2 for general $n$. The second subsection is concerned with another type of intersecting partition system, which generalizes 1-intersecting partition systems. 


\subsection{Towards a complete theorem for $t$-intersecting partition sys- tems}

Ahlswede and Khachatrian [3] have extended the Erdős-Ko-Rado theorem for set systems by determining the size and structure of all maximal $t$-intersecting set systems $P \subseteq\left(\begin{array}{c}{[n]} \\ k\end{array}\right)$ for all possible $n \leq(k-t+1)(t+1)$. This remarkable result went beyond proving a conjecture by Frankl [7] that stated a specific list of candidates for maximal set systems. Next, we state conjectures for uniform $t$-intersecting partition systems, which parallel the conjecture of Frankl and the theorem of Ahlswede and Khachatrian, respectively.

For $0<i \leq\lfloor(k-t) / 2\rfloor$, define the partition system

$$
\begin{aligned}
\mathcal{P}_{i}(n, k, t)= & \left\{P \in U_{k}^{n}: \mid P \cap\{[1, c],[c+1,2 c],\right. \\
& \ldots,[(t+2 i-1) c+1,(t+2 i) c]\} \mid \geq t+i\} .
\end{aligned}
$$

Note that $\mathcal{P}_{0}(n, k, t)=\mathcal{P}(n, k, t)$.

Conjecture 12. Let $n \geq k \geq t \geq 1$, and let $\mathcal{P} \subseteq U_{k}^{n}$ be a t-intersecting partition system. Then

$$
|\mathcal{P}| \leq \max _{0 \leq i \leq \frac{k-t}{2}}\left|\mathcal{P}_{i}(n, k, t)\right|
$$

Conjecture 13. Let $n \geq k \geq t \geq 1$, and let $\mathcal{P} \subseteq U_{k}^{n}$ be a $t$-intersecting partition system. If $n_{0}(c, t, i+1)<n<n_{0}(c, t, i)$, then $|\mathcal{P}| \leq\left|\mathcal{P}_{i}(n, k, t)\right|$. Moreover, this bound is tight if and only if $\mathcal{P}$ is equal (up to permutations on $[1, n]$ ) to $P_{i}(n, k, t)$.

One could hope to be able to use the ideas in [3] to prove these conjectures; however, key techniques such as left compression, which are used in their proofs, do not seem to work when dealing with partition systems.

We conclude with an infinite sequence of parameters $(n, k, t)$ for which $\mathcal{P}(n, k, t)$ is not maximal.

Proposition 14. Let $n>k>3$ and $t=k-3$. If $k>k_{0}(c)$, then $\left|\mathcal{P}_{1}(n, k, t)\right|>$ $|\mathcal{P}(n, k, t)|$.

Proof. Let $c=n / k$. It is not difficult to see that $|\mathcal{P}(n, k, k-3)|=\left(\begin{array}{c}3 c-1 \\ c-1\end{array}\right)\left(\begin{array}{c}2 c-1 \\ c-1\end{array}\right)$ and $\left|\mathcal{P}_{1}(n, k, t)\right|=(t+2)\left(\begin{array}{c}2 c-1 \\ c-1\end{array}\right)-t-1$. For $k$ (and $t$, since $\left.t=k-3\right)$ sufficiently large

$$
\left(\begin{array}{c}
3 c-1 \\
c-1
\end{array}\right)\left(\begin{array}{c}
2 c-1 \\
c-1
\end{array}\right)<(t+2)\left(\begin{array}{c}
2 c-1 \\
c-1
\end{array}\right)-t-1 .
$$




\subsection{Conjectures for partially t-intersecting partition systems}

P. L. Erdős and Székely [6] define another type of intersecting partitions, which we call here partially t-intersecting. Two partitions $P_{1}, P_{2} \in P_{k}^{n}$ are said to be partially $t$-intersecting if there exist two classes $C_{1} \in P_{1}$ and $C_{2} \in P_{2}$ such that $\left|C_{1} \cap C_{2}\right| \geq t$. The case $t=2$ is of particular interest: two partitions are said to intersect if their meet is above an atom.

Conjecture 15. (Czabarka's conjecture, see [6]) Let $n \leq 2 k-1$ and $\mathcal{P} \subseteq P_{k}^{n}$ be a partially 2 -intersecting partition system. Then, $|\mathcal{P}| \leq S(n-1, k)$. This bound is attained by the system

$$
\left\{P \in P_{k}^{n}:[1,2] \subseteq A \text {, for some } A \in P\right\} .
$$

We pose a similar conjecture for uniform partition systems.

Conjecture 16. Let $n \geq k$ and $t \leq c=n / k$. Let $\mathcal{P} \subseteq U_{k}^{n}$ be a partially $t$-intersecting partition system. Then, $|\mathcal{P}| \leq\left(\begin{array}{c}n-t \\ c-t\end{array}\right) U(n-c, k-1)$. Moreover, this bound is tight if and only if $\mathcal{P}$ is equal (up to permutations of $[1, n]$ ) to

$$
\left\{P \in U_{k}^{n}:[1, t] \subseteq A, \text { for some } A \in P\right\} .
$$

Note that Theorem 1 confirms Conjecture 16 for $t=c$.

\section{References}

[1] R. Ahlswede, N. Alon, P. L. Erdős, M. Ruszinkó and L. A. Székely. Intersecting systems. Combinatorics, Probability and Computing, 6:127-137, 1993.

[2] R. Ahlswede, N. Cai and Z. Zhang. Higher level extremal problems. J. Combinatorics, Information and System Sciences, 21(3-4):185-210, 1996.

[3] R. Ahlswede and L. H. Khachatrian. The complete intersection theorem for systems of finite sets. European J. Combin., 18(2):125-136, 1997.

[4] M. Deza and P. Frankl. Erdős-Ko-Rado theorem-22 years later. SIAM J. Algebraic Discrete Methods, 4(4):419-431, 1983.

[5] P. Erdős, C. Ko, and R. Rado. Intersection theorems for systems of finite sets. Quart. J. Math. Oxford Ser., 2(12):313-320, 1961.

[6] P. L. Erdős and L. A. Székely. Erdős-Ko-Rado theorems of higher order. Numbers, information and complexity (Bielefeld, 1998), 117-124, 2000.

[7] P. Frankl. The Erdös-Ko-Rado theorem is true for $n=$ ckt. Colloq. Math. Soc. János Bolyai, 18:365-375, 1978.

[8] A. Hajnal and B. Rothschild. A generalization of the Erdős-Ko-Rado theorem on finite set systems. J. Combinatorial Theory Ser. A, 15:359-362, 1973. 
[9] M. Simonovits and V. Sós. Intersection theorems for graphs. Problèmes combinatories et théorie des graphes, Colloq. Internat. CNRS, 260:389-391, 1978.

[10] M. Simonovits and V. Sós. Intersection theorems for graphs II. Colloq. Math. Soc. János Bolyai, 18:1017-1030, 1978.

[11] M. Simonovits and V. Sós. Intersection theorems on structures. Annals of Discrete Mathematics, 6:301:313, 1980.

[12] R. M. Wilson. The exact bound in the Erdős-Ko-Rado theorem. Combinatorica, 4(2-3):247-257, 1984. 